\title{
Antifungal Resistance of Candida Spp. in Bloodstream Infection
}

\author{
Truong Thien Phu*, Nguyen Ngoc Truong, Nguyen Thi Nam Phuong, Pham Huy Bup and Pham Thi \\ Ngoc Thao \\ Head of Microbiology Department, Cho Ray Hospital, Vietnam \\ *Corresponding author: Truong Thien Phu, Head of Microbiology Department, Cho Ray Hospital, Vietnam
}

\section{ARTICLE INFO}

Received: 幽 December 20, 2019

Published: January 07, 2020

Citation: Truong Thien Phu, Nguyen Ngoc Truong, Nguyen Thi Nam Phuong, Pham Huy Bup,Pham Thi Ngoc Thao. Antifungal Resistance of Candida Spp. in Bloodstream Infection. Biomed J Sci \& Tech Res 24(2)2020. BJSTR. MS.ID.004032.

Keywords: Candida Spp; Bloodstream Infection

\section{ABSTRACT}

Objective: To investigate the distribution of fungal species causing bloodstream infection, and their antifungal resistance.

Methods: The descriptive cross-sectional study was conducted at Cho Ray Hospital, Ho Chi Minh city, Vietnam in 2018. All blood culture samples of patients which were positive with yeast were analyzed. Blood samples were taken to BD Bactec bottles and monitored in Bactec 9120 (Becton Dickinson, USA). Sub-cultures were performed on Sabouraud agar after the bottles flagged positive. The growth obtained after overnight incubation. Identification and drug susceptibility testing were performed by YST card and AST YS07 card on Vitek 2 Compact system (BioMerieux, France) according to CLSI standard.

Results: There were 89 patients who showed fungal positive blood culture results. Three leading pathogens caused bloodstream infection were $C$. tropicalis $(31 ; 34.8 \%), C$. albicans $(20 ; 22.5 \%)$, C. glabrata $(18 ; 20.2 \%)$. Resistance of Candida spp. to Fluconazone was highest (8; 9.9\%), followed by Amphotericin B (7; 8.6\%), Voriconazole (7; 8.6\%), Flucytocine $(1 ; 1.2 \%)$. Caspofungin and Micafungin showed no resistance.

Conclusion: The most common fungi in bloodstream infection were $C$. tropicalis, C. albicans and C. glabrata, respectively. The resistance to antifungus of Candida spp. strains was less than $10 \%$. In this study, the resistance of Candida spp. to antifungal drugs was relatively low, however, it is necessary to continuously monitor the trend of drug resistance of fungal strains in order to detect changes in the resistant rates in order to handle appropriately.

\section{Background}

Candida spp. is a yeast that resides on the skin, in the gastrointestinal tract, urinary tract ... is the cause of opportunistic infection, causes disease in immunocompromised patients, patients treated with medical therapy and invasive surgery includes broadspectrum antibiotics, chemicals and organ transplants [1,2]. Candida spp. can thrive in the intestine, break down the intestinal wall and enter the bloodstream. There are many toxins of Candida in bloodstream infection, which are very dangerous for health and difficult to treat. Fungal infections of the bloodstream include several conditions such as Candida blood infection, endocarditis, meningitis and other forms of deep organ damages. Nearly $96 \%$ of fungal infections were caused by Candida spp. $[3,4]$. Mortality due to Candida has been reported up to $40-50 \%[5,6]$. Candida spp. is the top four causes of sepsis in hospitals, accounting for $9 \%$ of the total number of pathogenic microorganisms [7]. In India, according to the study of Vibhor Tak and colleagues, $43 \%$ of fatality was caused by fungal infections [2], in the study of Ying-Lien Chena et al, this rate was 40 to $70 \%$ [8]. In Vietnam, studies on fungal infections are limited and have not been paid attention like bacterial infections, with little data on antifungal drug resistance. In this study, we investigated the causative agents of antifungal blood infections and the situation of antifungal drug resistance. 


\section{Research Methods}

The descriptive cross-sectional study was conducted at Cho Ray Hospital, Ho Chi Minh city, Vietnam, from January 1, 2018 to December 31, 2018. All blood culture samples of patients which were positive with yeast were analyzed. Blood samples were taken in BD Bactec bottle and monitored in the Bactec 9120 machine (Becton Dickinson, USA). Sub-cultures were performed on Sabouraud agar after the bottles flagged positive. The growth obtained after overnight incubation. It was further identified by YST card and performed antifungal by AST YS07 card on Vitek 2 Compact system (BioMerieux, France). YST card can identify most Candida species, AST YS07 card detects susceptibility of 6 antifungal drugs: Amphotericin B, Caspofungin, Flucytosine, Fluconazole, Micafungin and Voriconazole according to CLSI standard.

\section{Results}

\section{Distribution of Fungal Species in Bloodstream Infection}

There were 89 patients who were positive for fungal blood culture. Candida tropicalis was the most common causative species (31; 34.8\%), followed by C. albicans (20; 22.5\%), C. glabrata (18; $20.2 \%)$, C. parapsilosis (8; 9.0\%), Cryptococcus neoformans (4; $4.5 \%)$, C. guilliermondii $(3 ; 3.4 \%)$, C. rugosa $(1 ; 1.1 \%)$, Kodamaea ohmeri $(1 ; 1,1 \%)$, Trichosporon asahii $(1 ; 1,1 \%)$, C. spherica $(1 ;$ $1,1 \%)$ and Trichosporon mucoides $(1 ; 1,1 \%)$ (Table 1$)$.

\section{Resistant Pattern of Candida Spp. in Bloodstream Infection}

Resistant rate of Candida spp. was highest to Fluconazole (8; 9.9\%), followed by Amphotericin B (7; 8.6\%), Voriconazole (7; $8.6 \%)$, Flucytosine (1; 1.2\%). Caspofungin and Micafungin showed no resistance (Figure 1).

Among 31 C. tropicalis, resistant isolates to Fluconazole and Voriconazole were 7 (22.6\%) and 7 (22.6\%).

Among 20 C. albicans, resistant isolates to Amphotericin B were 3 (15.0\%). Among 18 C. glabrata, resistant isolates to Amphotericin $\mathrm{B}$ and Fluconazole were $2(11.1 \%)$ and 1 (5.6\%), respectively.

\section{Discussion}

There were 89 patients who showed positive blood cultures with fungi in 2018 at Cho Ray Hospital. The three leading species of fungal infection are C. tropicalis (34.8\%), C. albicans $(22.5 \%)$ and C. glabrata (20.2\%), respectively (Table 1) while our data in 2017, the three leading agents of fungal infections were $C$. tropicalis (33\%), C. albicans (27\%) C. parapsilosis (14\%), and the fourth were $C$. glabrata (12\%). In recent years, $C$. tropicalis is the species with the highest proportion of Candida bloodstream infection in Cho Ray hospital. In other countries, C. tropicalis also accounted for the highest proportion of blood fungal infections, as in Taiwan, accounting for $41.9 \%$ [9], in India 39\% [2], however in other parts of the country in India, C. albicans accounted for the highest rate of $48.57 \%$ [10] while C. parapsilosis took the highest rate $(28.4 \%)$ in Italy [11]. The above results show that the distribution of fungal pathogens of bloodstream infection is different in vary geographical regions, even changes over the years. Resistant rate of Candida spp. in bloodstream infection was highest to Fluconazone (8; 9.9\%), followed by Amphotericin B (7; 8.6\%), Voriconazole (7; 8.6\%), Flucytocine $(1 ; 1,2 \%)$ [12]. Caspofungin and Micafungin showed no resistance (Figure 1).

Table 1: Distribution of fungal species in bloodstream infection.

\begin{tabular}{|c|c|c|}
\hline Pathogens & Number & Percent \\
\hline Candida tropicalis & 31 & $34.80 \%$ \\
\hline Candida albicans & 20 & $22.50 \%$ \\
\hline Candida glabrata & 18 & $20.20 \%$ \\
\hline Candida parapsilosis & 8 & $9.00 \%$ \\
\hline Cryptococcus neoformans & 4 & $4.50 \%$ \\
\hline Candida guilliermondii & 3 & $3.40 \%$ \\
\hline Candida rugosa & 1 & $1.10 \%$ \\
\hline Kodamaea ohmeri & 1 & $1.10 \%$ \\
\hline Trichosporon asahii & 1 & $1.10 \%$ \\
\hline C. spherica & 1 & $1.10 \%$ \\
\hline Trichosporon mucoides & 89 & $1.10 \%$ \\
\hline Total & & $100.00 \%$ \\
\hline
\end{tabular}

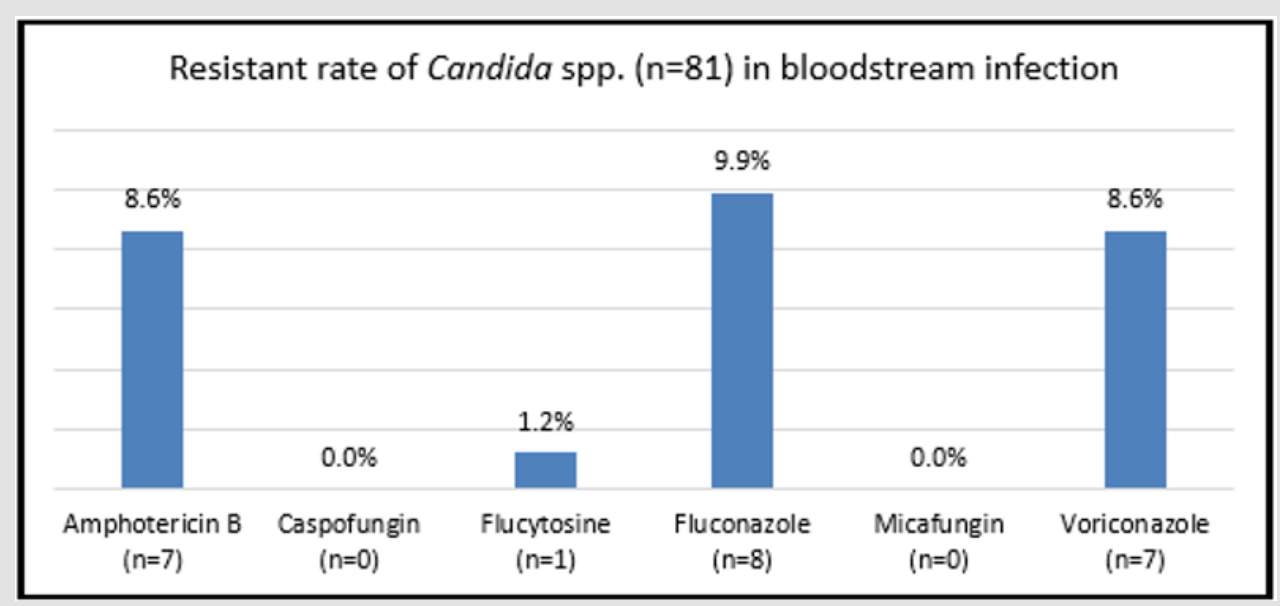

Figure 1: Resistance of Candida spp. in bloodstream infection. 
The resistant rate of $C$. tropicalis to antifungal drugs in bloodstream infections was relatively high, resistance to Fluconazole was $22.6 \%$, Voriconazole was also $22.6 \%$, these rates are double higher than the rates in a study of Xu H, Yu SY, Zhou ML, et al. at Hospital in Central China: among C. tropicalis isolates, resistance rates to fluconazole and voriconazole were $11.9 \%$ and $7.7 \%$, respectively (2012-1014) [1]. In the similar study in Taiwan, the resistance rate of $C$. tropicalis to Fluconazole was 13.9\% (20112014) [9]. The high frequency of $C$. tropicalis and increasing trend azole non-susceptibility requires noting. The resistance rate of $C$. albicans to Amphotericin B is alarming (15.0\%) because it is one of the main antifungal drugs for invasive candidemia and according to a study in China, C. albicans showed a wild type phenotype to Amphotericine B [1]. The resistant rates to C. glabrata of Amphotericine B and Fluconazole were 2 (11.1\%) and 1 (5.6\%), Voriconazole showed no resistant while in China resistant rates of Fluconazole and Voriconazole were $7,7 \%$ and $28.2 \%$, perhaps the usage of Voriconazole is limited in our hospital, the susceptibility is still high [13].

\section{Conclusion}

The common pathogens causing fungal infections in Cho Ray Hospital in 2018 are C. tropicalis, C. albicans, C. glabrata and C. parapsilosis. The resistance of Candida spp. was highest to Fluconazole, followed by Voriconazole and Amphotericin $B$. It is necessary to implement good hospital fungal infection control and strict antifungal management policies to help reduce fungal infections, resistance and death of patients due to fungal infections. This study provided local data of pathogen distribution and antifungal susceptibilities of invasive yeast species, which is essential for guiding antifungal treatment [14].

\section{References}

1. Xu H, Yu SY, Zhou ML, Ning YT, Xiao M, et al. (2019) Epidemiology and Antifungal Susceptibility Patterns Of Invasive Fungal Infections From 2012 To 2014 In A Teaching Hospital In Central China. Infect Drug Resist 12: $3641-3651$

ISSN: 2574-1241

DOI: 10.26717/BJSTR.2020.24.004032

Truong Thien Phu. Biomed J Sci \& Tech Res

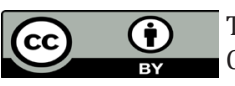

This work is licensed under Creative

Commons Attribution 4.0 License

Submission Link: https://biomedres.us/submit-manuscript.php
2. Tak V, Mathur P, Varghese P, Gunjiyal J, Xess I, et al. (2014) The Epidemiological Profile of Candidemia at an Indian Trauma Care Center. J Lab Physicians 6(2): 96-101.

3. Chakrabarti A, Chatterjee SS, Shivaprakash MR (2008) Overview of opportunistic fungal infections in India. Jpn J Med Mycol 49: 165-172.

4. Wisplinghoff H, Bischoff T, Tallent SM, Seifert H, Wenzel RP, et al. (2004) Nosocomial bloodstream infections in US hospitals: Analysis of 24,179 cases from a prospective nationwide surveillance study. Clin Infect Dis 39: 309-317.

5. Enoch DA, Ludlam HA, Brown NM (2006) Invasive fungal infections: A review of epidemiology and management options. J Med Microbiol 55: 809-818.

6. Badiee P, Hashemizadeh Z (2014) Opportunistic invasive fungal infections: Diagnosis \& clinical management. Indian J Med Res 139(2): 195-204.

7. Nucci M1, Colombo AL (2007) Candidemia due to Candida tropicalis: clinical, epidemiologic, and microbiologic characteristics of 188 episodes occurring in tertiary care hospitals. Diagn Microbiol Infect Dis 58(1): 77-82.

8. Chen YL, Yu SJ, Huang HY, Chang YL, Lehman VN, et al. (2014) Calcineurin Controls Hyphal Growth, Virulence, and Drug Tolerance of Candida tropicalis. Eukaryotic Cell 13(7): 844-854.

9. Wu PF, Liu WL, Hsieh MH, Hii IM, Lee YL, et al. (2017) Epidemiology and antifungal susceptibility of candidemia isolates of non-albicans Candida species from cancer patients. Emerging Microbes \& Infections 6(10): e87.

10. Bhattacharjee P (2016) Epidemiology and antifugal susceptibility of candida special in a tertiary care hospital, Kolkata, India. Curr Med Mycol 2(2): 20-27.

11. Bassetti M, Taramasso L, Nicco E, Molinari MP, Mussap M, et al. (2011) Epidemiology, species distribution, antifungal susceptibility and outcome of nosocomial candidemia in a tertiary care hospital in Italy. PLoS One 6: e24198.

12. Guinea J (2014) Global trends in the distribution of Candida species causing candidemia. Clin Microbiol Infect 20(Sup 6): 5-10.

13. Shin JH (2017) species distribution and antifugal susceptibilities candida clinial isoates from 14 tertiary hospitals in Korea. International Jounal of Antimicrobial Agents 5051 s163.

14. Krishna S, Sudheer A, Dinesh K, Kumar H, Varma AK, et al. (2015) Kodamaea (Pichia) ohmeri: Emerging yeast in diabetic foot and bloodstream infections. International Journal of Diabetes in Developing Countries. 35(3): 381-381.

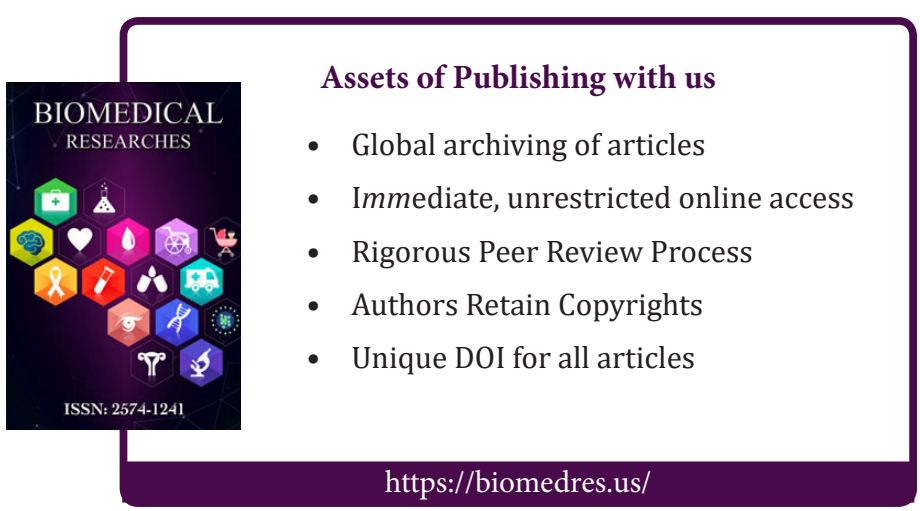

Copyright@ Truong Thien Phu | Biomed J Sci \& Tech Res | BJSTR. MS.ID.004032. 